\title{
Approach for the development of multi-agent system for future energy efficient transportation system employing smart grids \& sustainable energy
}

\author{
Ira Savitri, Ignatius Rendroyoko
}

PLN Indonesia, Jakarta and 12160, Indonesia

\begin{abstract}
Driven by the increasing of greenhouse gases (GHGs) from the utilisation of fossil fuels, particularly in transportation sector, and the shortage of fossil fuels itself, the development of future transportation system utilises electricity would be necessary. This development would significantly increase demand in power systems. Moreover, differences in characteristics, technologies and behaviour of electric vehicles users make it difficult to manage the demand and the efficiency of electricity use. Hence, an intelligent power system, smart grid system, would be needed to address these issues. Besides, implementing smart grids is expected to be a method to move toward utilisation of sustainable energy resources. However, despite many advantages promised by smart grid implementation, sophisticated criteria for steady and reliable power and transportation system challenges the implementation of smart grid. Therefore, in order to achieve future energy efficient transportation systems employing smart grids and sustainable energy, an organized approach is required. Thus, this paper describes an approach to develop multi-agent model which is expected to intelligently organise all the data and information, analyse and act based on the information in order to establish future energy efficient transportation system using smart grids and sustainable energy.
\end{abstract}

Keywords: Grid vehicles, smart grids, multi-agents, sustainable energy

\section{Introduction}

It is difficult to exaggerate the significance of electricity to support the development of future transportation system. As the increasing of Green House Gases (GHGs) is considerable from the utilisation of fossil fuels, particularly in transportation sector, and the shortage of fossil fuels itself, the development of electricity in transportation system is required. It is forecasted that future transportation systems would require much more electricity which might not be satisfied by conventional electricity systems. The utilisation of electric vehicles would rapidly increase demand in power systems. Moreover, differences in characteristics, technologies and behaviour of electric vehicle users make it difficult to manage the efficiency of electricity use. These reasons could encourage the interconnection of electric vehicles to an intelligent grid system or to smart grids.

Smart grids provide an opportunity to integrate electric vehicles to grids which is commonly known as vehicle-to-grid (V2G). The vehicles might include Plug-in Hybrid Electric Vehicles (PHEVs) and Electric Vehicles (EVs) [1]. The interconnection would enable bidirectional power flow between the grid and costumers (vehicles). These types of vehicles are commonly known as Gridable Vehicles (GVs). Smart grids also provide an opportunity to supply energy from renewable energy resources [2]. Thus, efforts of reducing GHGs emission by utilisation of electric vehicle can also be supported from power generation side by utilising sustainable energy resources that produce less or no GHGs emission.

Manuscript received June 25, 2015; revised September 16, 2015.

Corresponding author. Tel.: +62811-570 7730; E-mail address: ira.savitri@pln.co.id

doi: 10.12720 /sgce.4.4.346-354 
However, despite many advantages promised by smart grids, its implementation faces challenges on how to make the system steady and reliable. Hence, in order to achieve the future energy efficient transportation system employing smart grid and sustainable energy and also due to complexity of challenges and criteria that should be satisfied, an organized approach is required. Thus, this paper has a purpose to describe an approach to develop multi-agent model which is expected to intelligently organise all the data and information, analyse and act based on the information in order to establish future energy efficient transportation system using smart grids and sustainable energy. It will define the development of future transportation system as well as barriers and criteria or requirements that should be fulfilled to develop the system. It will also describe how smart grids are significant for that development. The stages of the approach for developing multi-agent system will also be outlined.

\section{The Development of Future Transportation System}

The development of future transportation system might not be far from the issue of energy efficiency and environmentally-friendly vehicles. The utilisation of electric vehicle which is non-polluting and super-efficient could be an answer for this issue [3]. It is found that over than $95 \%$ of motorized transports are dependent on oil. It is predicted that in 2030, if the trend is the same, the emission of GHGs from transportation sector would increase up to 9,000 megaton of carbon dioxide $\left(\mathrm{CO}_{2}\right)$ [4]. By the utilisation of electricity in future transportation system, adverse consequences of GHGs emission could be alleviated.

\subsection{PHEVs and EVs}

PHEVs and EVs are projected to be an answer for these environmental challenges. PHEVs are hybrid electric vehicles that use particular additional chargers to draw and storage energy from electricity grids and supply them to vehicles, a specification that differentiates them from conventional hybrid electric vehicles [5]. Using plug-in hybrid to replace petroleum energy to multi-source electrical energy, PHEVs could substantially reduce the production of $\mathrm{CO}_{2}$ [6]. Therefore, PHEVs can be considered as environmentally-friendly future vehicle.

For future model of electric vehicles, PHEVs offer more advantages than EVs. PHEVs can be run using gasoline or electricity [7]. Combustion engines are utilised to obtain chemical energy. Thus, if the electricity stored in a battery is almost gone or finished, the car still can be run using gasoline. Besides, if electricity price rises, PHEVs have an alternative to use gasoline. This advantage is not found in EVs. Therefore, the utilisation of PHEVs might provide better performance, higher efficiency and lower cost compared to EVs.

PHEVs will utilise the capability of V2G for charging batteries as well as acting as small power plants. These GVs can become loads and also energy resources. This technology enables bidirectional power flow between GVs' batteries and electricity power grids [8]. Energy can flow to or from GVs. Energy can be supplied from GVs to grids when there is under capacity of power in the grids while GVs are in condition of full capacity. It also can be conducted when market prices of electricity are high and GVs want to sell their electricity. This bidirectional power flow might benefit not only the customers (vehicles), but also grids.

\subsection{Challenges}

Besides benefits, the development of future transportation system might also face challenges. It is projected that several barriers such as increasing peak load and the uncertainty of power demand would challenge the development of this future transportation system. As GVs increase, power demand would also increase. There would be surges of demand in unpredictable time since GV users might charge batteries at uncertain time. Hence, the stability of power grids could be disturbed.

Another challenge could be related to developing facilities and infrastructure for future transportation system. Future electric vehicles might require appropriate socket or plug-in, charging places, remote meter reading, real time billing system, telecommunication tools and storage system [9]. Moreover, 
electric vehicle users might also put some ideal criteria for their future cars such as comfortable and safe, less or zero pollution, complete navigation system, recyclable and long lifetime, low maintenance cost, etc.

Furthermore, the development of future transportation system would face requirements for utilization of sustainable energy. The electricity from grids should mainly come from renewable energy in order to create environmentally-friendly power grids. The intermittency of renewable energy sources should be overcome using appropriate technologies.

Future transportation system should be built by addressing all the challenges and considering requirements for sustainable, steady and reliable transportation system, including power systems.

\section{Why Smart Grids for Future Transportation System?}

Complex challenges and criteria for efficient and sustainable power systems for future transportation lessen the capability of conventional grids to supply appropriate electricity. The need of electricity network that can intelligently respond to all users that are connected to it significantly increases [10]. The grids that use application of information and technology to manage large scale of renewable and decentralized generation to achieve sustainable, efficient and secure electricity supply, or smart grid [11], could be a solution to address the issue. The grid collaborates energy sources and users in a system by using technology that enables exchange of information and automatic intelligent responses.

There are several reasons for interconnection of future transportation system to smart grids. The first reason is significant increase of electricity demand due to massive development of electric vehicles in the next few years [12]. Thus, power grids need to consider that this change may result in increasing peak load and additional power generation. The second reason is the uncertainty of peak load due to a dynamic electricity demand with respect to time, location and users' behaviour. The unexpected demand could be the result of an inaccurate prediction of demand [7]. The demand could be an effect of an uncontrollable charging. GV users might charge batteries at different time. This behavior would volatile the power demand. With the absence of load management, this volatility would likely have a de-stabilizing effect on the grid. Using smart grid, it is expected that the information of this unpredictable demand could be responded automatically by energy sources.

The following reason to employ smart grids for supporting future transportation system is to achieve effective management of renewable energy in smart grid. The utilisation of smart grids provides advantages of reducing environmental impact from overall electricity supply [10]. Smart grids could be managed so that renewable energy sources become the priority to serve electricity demand.

Smart grids also present an opportunity for consumers to be more efficient in using electricity by receiving the information and choosing time to buy electricity. Moreover, this grid enables customers to sell electricity to the grid [13]. Hence, customers could manage their electricity utilisation and their expenses.

\section{Approach for the Development of Multi-Agent System}

The need to satisfy criteria and addressing challenges for developing future transportation system employing smart grids and the consideration to use sustainable energy drives the development of multiagent system application. This application might become an alternative that can provide an intelligent and automated monitoring function in the system [2]. Multi-agent system has an ability to analyze, reactively and pro-actively react and make a decision based on chosen criteria and requirements. It consists of independent software that has several characteristics as follows [14]:

- Autonomy, which means that each agent will operate in unsupervised mode and conduct diagnosis while changing its behaviour as required.

- Social ability, which means that each agent can operate, communicate with other agents, support data and information exchange and conduct negotiation.

- Reactivity, which means that the agent has an ability to react by the changing of its surroundings. 
- Pro-activeness, which means that the agent proactively taking an action to address problems and ensure that the information delivered is correct or the control activity done is required.

The approach to develop a multi-agent model incorporates several stages. These stages would be a circle stages since the criteria or requirements would change or improve from time to time (see Fig. 1).

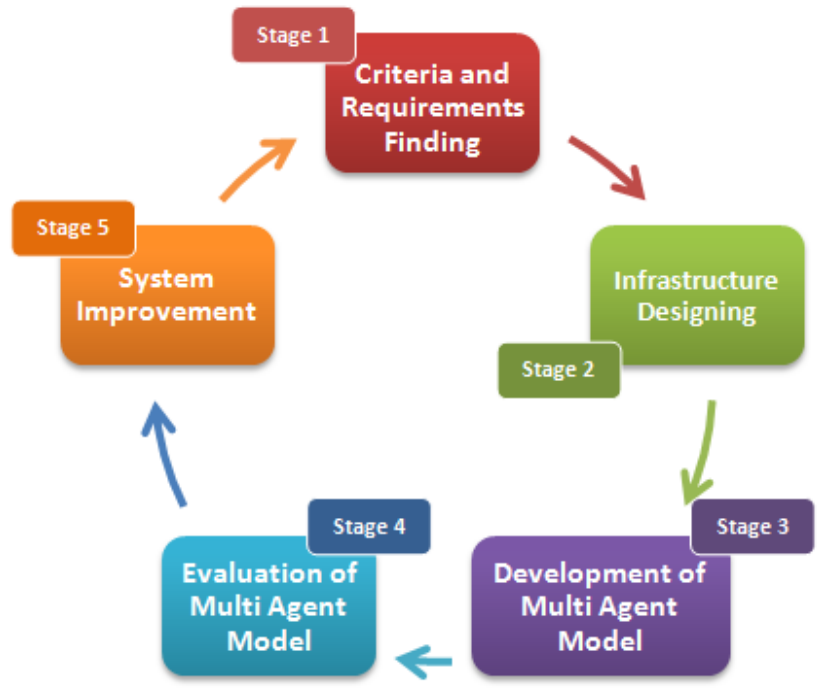

Fig. 1. Stage of multi-agent development.

\subsection{Stage 1-Criteria and requirements finding}

Finding the criteria or requirements for developing future transportation system and smart grids is the initial stage of multi-agent model development. The criteria or requirements can be differentiated into the criteria for ideal transportation system, which include GVs, transport facilities and infrastructure, and the criteria for smart grids. Some of the criterias for ideal GVs could be:

- GVs with parallel designs. These designs are favoured due to lower fuel economy, lower efficiency and higher component costs of series design.

- GVs with efficient use of energy and low emission. It is expected that the performance increase as the increasing capacity of energy storage.

- GVs with an advanced technology of batteries. High performance, long lifetime and low lifecycle cost batteries are preferably.

- GVs that can be functionally and continuously driven for relative long distance without repeatedly short time external recharging.

Another requirement for ideal GVs might be related to the ability to gather and update the information about real-time electricity prices. In multi-agent model, these future vehicles could also be equipped with smart meter and decision making instruments. Hence, GVs as one type of agents can react based on the information whether to buy electricity (recharge the battery) or sell electricity to the grid.

For smart grids, some criteria that should be fulfilled are efficiency, economic, reliability, sustainability and environmentally-friendly. The criteria of efficiency on grids could be achieved by reducing transmission and distribution electrical losses. It will give the advantage of more efficient power production. It will also improve asset utilisation investments that lead to ease the cost. Efficiency on grids can be conducted by maintaining standard voltage, controlling transformer, transmission and distribution loading. The efficiency would result in economic benefit. It is expected that by applying multi-agent system in smart grids, an optimal operation cost can be achieved. An economic grid operates under the basic laws of supply and demand, resulting in fair prices and adequate supplies. The economical grids can be obtained by optimal configuration network and dispatching of power plants. 
Another criteria for an ideal grid is reliability of the system. This criteria could be obtained by fulfilling some requirements such as sufficient reserve capacity of power plants that can serve an unpredictable increase of load and adequate transmission and transformer infrastructure.

In smart grids, the utilisation of sustainable energy would be an obligation. Sustainable energy is the provision of energy that meets the needs of the present without compromising the ability of future generations to meet their needs. Smart grids using multi-agent model should be able to maintain sustainability of energy in term of energy resources, energy losses and energy consumed.

In order to achieve environmentally-friendly grids, the utilisation of renewable energy sources would be significant. This utilization would substantially reduce the emission of GHGs. However, renewable resources present several challenges related to the grid reliability. The side effects to the grid reliability can happen due to following factors [9]:

- Low capacity factor and high variability cannot fulfill demand profile due to steeper net demand profile

- Low ability to fix load profile, particularly in case of solar wind

- High probability of forecast error for longer horizon.

- Congestion issues at transmission and distribution level due to spreading resources

These side effects should be a consideration in developing multi-agent model for grid systems. The utilization of large scale batteries or buffer generator and adequate transmission and distribution infrastructure might alleviate the effects.

\subsection{Stage 2-Infrastructure designing}

After reviewing the criteria and requirements for developing future transportation and smart grids system, the next stage of the approach would be designing the infrastructure. Several steps for designing the infrastructure are showed in Fig. 2.

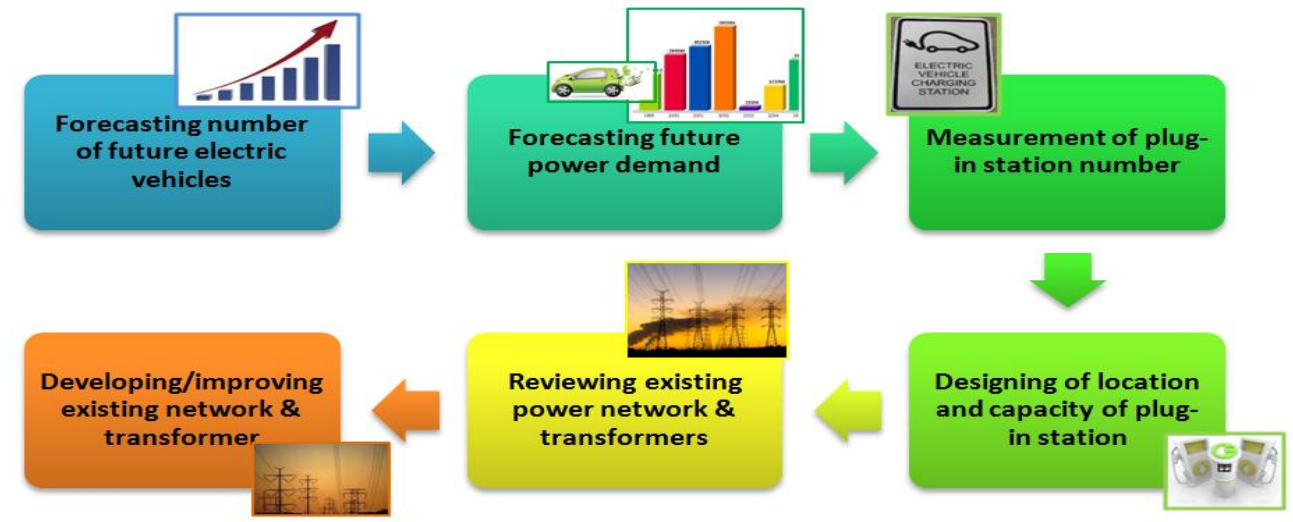

Fig. 2. Infrastructure designing.

To begin with, the first step is forecasting number of future electric vehicles. By considering requirements of future transportation and the increasing tendency of using environmentally-friendly vehicles, number of electric vehicles can be forecasted. Number of public and private electric vehicles will significantly increase as the increasing of people's awareness of sustainable energy and environmentally-friendly energy sources. After projecting number of future electric vehicle, the next step is forecasting power demand. Differences in technology from vehicle industries might lead to different characteristic of vehicles [5]. Some vehicles use more efficient electricity than others. The efficiency could be obviously observed from storage or battery technology. The technology could influence lifetime of batteries and time of charging required.

The following step is measuring plug-in station needed. Plug-in station number can be calculated after number of vehicles and power demand measured. The number can be different in each area/location 
depend on the density of GVs used in that location. The following step to develop infrastructure designing for plug-in station and smart grids is determining the location and capacity of plug-in stations. The location of plug-in station will affect the capacity of it. The location that is predicted will be crowded will have more plug-in stations or higher capacity of plug-in stations. After the location and capacity of plugin stations are decided, reviewing of existing power network and capability of transformers is required. It could be that some locations of plug-in are not supported by sufficient of power network or transformer. Hence, an augmentation of power network or transformer is needed. Additional network or transformers might be needed in some location of plug-in station where the existing network and transformers do not support that.

\subsection{Stage 3-Develop multi-agent model}

The development of the multi-agent models is the following stage the approach. Each agent has certain roles and abilities. All of the agents will support each other in term of providing information, exchanging data and conducting negotiation. In this multi-agent model, there are six main agents that can be chosen for future transportation and smart grid systems; User Agent (UA), Control Agent (CA), Power Plant Agent (PPA), Aggregation Agent (AA), Dispatch Agent (DA) and Database Agent (DBA).

UA is electric vehicles, PHEVs or EVs. UA has an ability to connect users or vehicles to smart grids. This agent is capable of receiving real time information and presenting all relevant information to users, such as information about real time electricity price which is significantly useful for users to make a decision to sell or to buy the electricity in a certain time. The development of this agent has two main goals. The first goal is to ensure that vehicles have enough electricity power from initial place of its departure location to the destination or to nearest plug-in station. The other goal is to provide information to other agents related to location of charging, battery condition and other important information required by the system. This information is substantial for predicting the time and location of vehicles conducting recharging. Moreover, this information is also useful for evaluating the demand forecast and revising the load curve. It would enable fast response to demand changes and preparation for unexpected demand. For maintaining communication with other agents, UA can be facilitated with telecommunication tools, such as global system for mobile communication (GSM).

The next agent in the model is CA. This agent has an ability to monitor reliability of the system. CA will monitor system voltage and transformer loading in every substation, loading of transmission and distribution lines, frequency, and check the status of any instrument that is in maintenance or is taken out from the system. The information recorded in CA will be transferred to DA. In DA, the data will be used to sort power plants and make a dispatch order based on forecasted demand and to obtain optimal network configuration that satisfy reliability criteria.

The following agent is PPA. This agent is responsible for storing distributed energy resources information as well as monitoring and controlling power level and connection status to the system. The information might contain identification number, location, electricity price, availability of power plants, type (solar cells, micro turbines, fuel cell and other power plants), power rating $(\mathrm{kW})$, cost function and quantity of power produced. The information from PPA will be used by AA; another agent in the multiagent model. AA has a role to receive the information from PPA regarding the bids of electricity price and aggregate the supply into different price slots depending on the bid prices provided by PPA.

All the data that present real time information received from UA, CA and AA will be collected by DA. This agent offers a dynamic contact point through which they can share and retrieve bidding information. The dispatch process will be conducted by this agent. DA will analyze and produce optimal solution of network configuration that is efficient, economic, reliable and environmentally-friendly. The information of real time electricity price will be sent to UA for a consideration of buying or selling electricity. This information presents the opportunity for vehicle users to decide time of charging for more economic cost and time to sell the electricity to power grids for receiving benefit from higher electricity selling price. Another agent in the model, DBA, has an ability to collect and store all the information of electricity price, demand and bidding in a database. It can also provide an access to the database for other agents. 
The interaction and process in multi-agent model that is developed can be seen in the flowchart in Fig. 3. The information and communication flows are also showed in the flowchart. The process is described as follow; on the side of power generation (owner), negotiation price which contain ID, type of generation, price, availability and quantity of power produced will be provided by PPA. PPA will send the data to AA where the data will be processed to generate price tiers. Price tiers will be sent back to PPA. On the other side, CA will provide information to DA regarding the system condition which is associated with loading on network and transformers, maintenance schedule for network and transformers, system voltage, frequency and losses. The data from AA and CA as well as forecasted demand will be processed by DA. The dispatch result must fulfill criteria that are previously required, such as efficient, economic, reliable, sustainable energy and environmentally-friendly. After dispatching, the electricity price will be informed to customers through UA (Vehicle). UA will receive the offered electricity price and thus make decision for buying or not buying. If UA conduct buying /battery charging through providers of plug-in stations (also known as EV supply equipment or EVSE), UA will send data of electricity purchasing, charging location and battery condition to DA. It will conduct update dispatch.

The result of the dispatch will be sent to AA and CA. AA will send the dispatch data to chosen PPA. CA will conduct monitoring and maintaining system condition, such as system voltage, frequency, loading and losses. PPA chosen will conduct power and voltage compliance with the standard required.

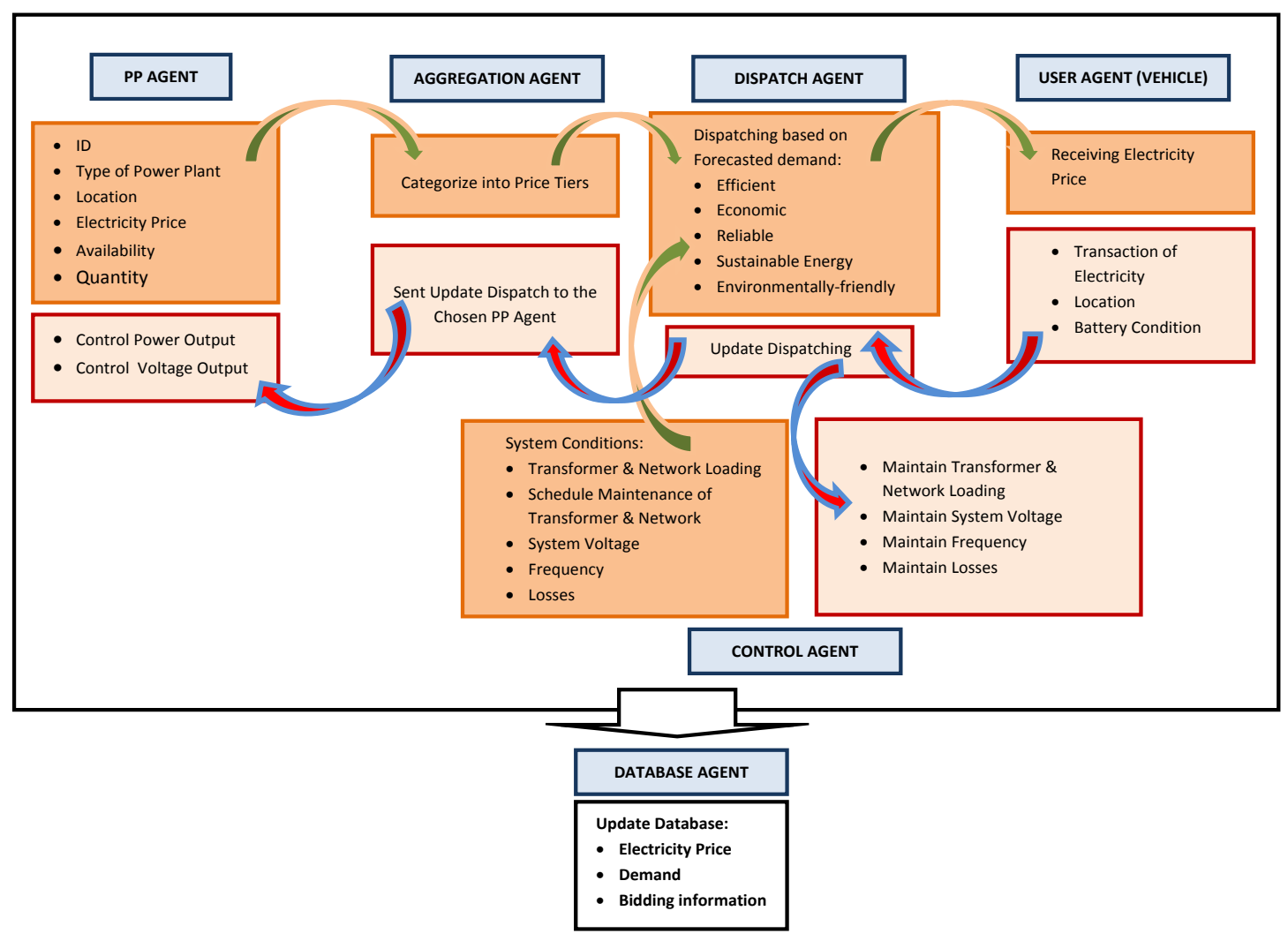

Fig. 3. Multi-agent flow chart.

\subsection{Stage 4-Evaluation of multi-agent model}

This stage is about the evaluation of multi-agent model developed in the previous stage. It will analyse flaws or the area for improvement of the model. The data about system condition and process in transportation and smart grids is stored in Data Base Agent. This record can be a consideration for evaluating the system, whether the system run as it is required and fulfill all the criteria or not. 


\subsection{Stage 5- System improvement}

The final stage of the approach is system improvement. There might be many new criteria or requirements as a new transportation system is developed and smart grids is established. After evaluating the system, it might have some weaknesses or areas of improvement that should be satisfied. The improvement of the model will be continually required for compliance of existing condition or forecasting of future condition.

\section{Conclusions}

Future transportation system will tend to utilise much more electricity. Driven by the effort to reduce environmental impacts, sustainable energy resources will be required. Moreover, significant increase and unpredictable characteristics of electricity use in the future transportation system will affect the ability of electricity system to supply power. Thus, interconnection with smart grids tends to be substantial. Considering that smart grids uses renewable resources, the intention to improve environment can be achieved. For managing efficient energy of future transportation system employing smart grids and sustainable energy, multi-agent system can be utilised.

Several stages can be conducted to develop the system. The stage begins with finding criteria and requirements for future transportation system and smart grids and is followed by designing the infrastructure of plug-in location and measuring the availability of power network. The development of multi-agent model is the next important step. The evaluation and improvement of the model will be the next stage of the approach. By implementing the multi-agent system, it is expected that the objective can be achieved. For the improvement of the model, it is recommended that there is corporation among governments, power utility providers and vehicle industries since the installation of plug-in station and the development of smart grids will influence the urban planning and also the residents staying near by the location. Sufficient information and socialization given to residents will be required to avoid residents' rejection.

\section{References}

[1] Mullen SK. Plug-in hybrid electric vehicles as a source of distributed frequency regulation. PhD dissertation. Department of Electrical Engineering, Minnesota University. Minnesota, United States; 2009.

[2] Pipattanasomporn M, Feroze H, Rahman S. Multi-agent systems in a distributed smart grid: Design and implementation. In: Proc. IEEE/PES Power Systems Conference and Exposition, 2009:1-8.

[3] Grob GR. Future Transportation with smart grids \& sustainable energy. In: Proc. 6th International Multi-Conference on Systems, Signals and Devices, 2009:1-5.

[4] Woodcock J, Banister D, Edwards P, Prentice AM, Robert I. Energy and transport. The Journal of Energy \& Health Series, 2007; 370(10):78-88.

[5] Bradley TH, Frank AA. Design, demonstrations and sustainability impact assessments for plug-in hybrid electric vehicles. Renewable and Sustainable Energy Review, 2007; 13(1):115-128.

[6] Saber AY, Venayagamoorthy GK. Plug-in vehicles and renewable energy sources for cost and emission reductions. IEEE Transactions on Industrial Electronics, 2010; 58(4):1229-1238.

[7] Waraich RA, Galus MD. Dobler C, Balmer M, Andersson G, Axhausen KW. Plug-in hybrid electric vehicles and smart grid: investigations based on a micro-simulation. Presented at: The 12th International Conference on Travel Behaviour Research, 2009.

[8] Kempton W, Udo V, Huber K, Komara K, Letendre S, Baker S, Brunner D, Pearre N. A test of vehicle-to-grid (V2G) for energy storage and frequency regulation in the PJM system. Research report. Industry-University Research Partnership, 2011.

[9] Moslehi K, Kumar R. Smart grid-A reliability perspective. IEEE Transactions on Smart Grid, 2010; 1(1):57-64.

[10] Belmans R. (2010). Smart grids, strategy deployment document for Europe'S electricity network of the future, European technology platform. [Online]. Available: http://www.smartgrids.eu/documents/sra/sra_finalversion.pdf

[11] Hommelberg MPF, Warmer CJ, Kamphuis IG, Kok JK, Schaeffer G. Distributed control concepts using multi-agent technology and automatic market: an indespensable feature of smart power grids. In: Proc. IEEE Power Engineering Society General Meeting, 2007:1-7. 
[12] Kamboj S, Kempton W, Decker KS. Deploying power grid-integrated electric vehicles as a multi-agent system. In: Proc. 10th International Conference on Autonomous Agents and Multiagent Systems - Innovative Applications Track, 2011:13-20.

[13] Cleveland CJ, Morris C. Dictionary of Energy Elsevier. Amsterdam; 2006.

[14] McArthur SDJ, Hossack JA, Jahn G. Multi-agent system for diagnostic and condition monitoring applications. Power Engineering Society General Meeting, 2003; 1(1):48-51. 